\title{
电沉积时间对 $\mathrm{SiO}_{2}-\mathrm{ZnO}$ 复合光子晶体形貌及 光学特性的影响
}

\author{
间刚印，张欣，黄鹏，王垒，戚芳，冯博学* \\ 兰州大学物理科学与技术学院, 教育部磁学与磁性材料重点实验室, 兰州 730000 \\ * 联系人, E-mail: fengbx@1zu.edu.cn
}

2009-11-30 收稿, 2010-07-29 接受

国家自然科学基金(60576013, 60536010, J0630313)和甘肃省自然科学基金(096RJZA054)资助项目

\begin{abstract}
摘要 采用垂直沉积法成功制备了 $\mathrm{SiO}_{2}$ 光子晶体薄膜. 以 $\mathrm{SiO}_{2}$ 光子晶体薄膜做模板, 采用电沉积 和烧结处理在 ITO 基底上制备了 $\mathrm{SiO}_{2}-\mathrm{ZnO}$ 复合光子晶体. 利用扫描电子显微镜观察了 $\mathrm{SiO}_{2}$ 光子 晶体模板及不同沉积时间所制备的 $\mathrm{SiO}_{2}-\mathrm{ZnO}$ 复合光子晶体薄膜形貌. 研究发现, 当沉积时间较 短时, $\mathrm{ZnO}$ 颗粒随机生长在 $\mathrm{SiO}_{2}$ 微球表面. 随着沉积时间的延长, $\mathrm{ZnO}$ 颗粒均匀地生长在 $\mathrm{SiO}_{2}$ 微 球表面, 以至于 $\mathrm{ZnO}$ 颗粒完全掺入模板的间隙. 对所制备的光子晶体薄膜进行反射谱测量, 发现 在膜法线方向上, 光子晶体薄膜有光子带隙的出现.
\end{abstract}

关键词

光子晶体

电沉积

光子带隙
1987 年 $\mathrm{John}^{[1]}$ 与 Yablonovitch ${ }^{[2]}$ 提出用光子晶体 控制光线传播和抑制自发辐射之后, 这种光子带隙 材料的研究吸引了越来越多科学工作者的注意. 光 子晶体是一种介电常数周期性调制的有序结构. 由 于布拉格散射，这种周期性排布结构具有光子禁带 窗口也称为光子带隙. 采用自组装胶体微球来制备 在光波范围内具有光子带隙的三维光子晶体有其无 可比拟的优势 ${ }^{[3]}$, 这种胶体晶体也称为蛋白石.

近来, 自组装制备具有蛋白石结构的光子晶体 出现了很多种方法, 如垂直沉积法 ${ }^{[4]}$ 、双基片垂直沉 积法 ${ }^{[5]}$ 、对流沉积法 ${ }^{[6]}$ 、电泳沉积法 ${ }^{[7]}$ 等. 尽管如此, 因为在自组装的过程中会生成许多种内生缺陷, 如 面缺陷、线缺陷、点缺陷等, 三维蛋白石光子晶体的 制备工艺仍存有技术性困难. 在自组装制备过程中, 胶体球的表面电荷对成功制备出高质量的光子晶体 有很大影响, 一些科学工作者采用丁二酸对 $\mathrm{SiO}_{2}$ 胶 体球进行改性来提高其表面电荷密度并制备出了高 质量的 $\mathrm{SiO}_{2}$ 光子晶体 ${ }^{[8]}$. 因垂直沉积方法十分简单, 得到的光子晶体质量高, 因而本文中, 我们采用垂直 沉积法自组装制备 $\mathrm{SiO}_{2}$ 光子晶体模板.
理论计算表明, 由于二氧化硅(折射率 $n=1.47$ )和 聚合物蛋白石其折射率较小, 尽管在(111)方向上出 现光子带隙, 但不具备完全光子带隙. 为了制备完全 光子带隙光子晶体, 越来越多的科学工作者以蛋白 石结构为模板掺杂不同的材料制备复合结构的光子 晶体以及反相蛋白石光子晶体 ${ }^{[9 \sim 11]} . \mathrm{ZnO}$ 是一种具有 宽带隙电子结构的半导体材料, 它具有相对其他材 料较高的折射率 (2.1), 一些研究者利用不同的方 法 ${ }^{[12,13]}$ 制备出了不同的 $\mathrm{SiO}_{2}-\mathrm{ZnO}$ 复合光子晶体结构.

本文采用电沉积法和烧结 $\left(500^{\circ} \mathrm{C}, 2 \mathrm{~h}\right)$ 处理法制 备出 $\mathrm{SiO}_{2}-\mathrm{ZnO}$ 复合光子晶体. 在电沉积过程中, 选 择了不同的沉积时间来制备 $\mathrm{SiO}_{2}-\mathrm{ZnO}$ 复合光子晶体.

\section{1 实验部分}

(i ) 实验所需原材料. 去离子水(分析纯); 无 水乙醇(分析纯); 正硅酸乙酯(TEOS, 分析纯); 浓氨 水 (25\% 28\%, 质量分数); 二水醋酸锌 (分析纯).

(ii) $\mathrm{SiO}_{2}$ 胶体球的制备. 采用 Stöber 方法 ${ }^{[14]}$ 制 备 $\mathrm{SiO}_{2}$ 胶体球, 首先将 $3.0 \mathrm{~mL}$ 去离子水与 $7.0 \mathrm{~mL}$ 浓 氨水混合加人玻璃反应容器中, 再加人含 $4.8 \mathrm{~mL}$ 
TEOS 与 $50 \mathrm{~mL}$ 无水乙醇的溶液, 在 $30^{\circ} \mathrm{C}$ 恒温水浴中 磁力搅拌 $12 \mathrm{~h}$. 其化学反应过程包括水解与缩合两 个过程，以下是其反应方程式:

$$
\begin{gathered}
\mathrm{Si}\left(\mathrm{OCH}_{2} \mathrm{CH}_{3}\right)_{4}+4 \mathrm{H}_{2} \mathrm{O}=\mathrm{Si}(\mathrm{OH})_{4}+4 \mathrm{C}_{2} \mathrm{H}_{5} \mathrm{OH} \\
n \mathrm{Si}(\mathrm{OH})_{4}=n \mathrm{SiO}_{2}+2 n \mathrm{H}_{2} \mathrm{O}
\end{gathered}
$$

反应完毕后对所制备的 $\mathrm{SiO}_{2}$ 胶体球反复离心洗 涤，超声分散 6 次以上以去除反应物残余.

(iii) $\mathrm{SiO}_{2}$ 光子晶体模板的制备. 采用垂直沉积 法制备 $\mathrm{SiO}_{2}$ 光子晶体模板 ${ }^{[4]}$, 制备过程如下: 依次用 丙酮, 乙醇, 二次蒸馏水对 ITO 导电玻璃 $(25 \mathrm{~mm} \times 10$ $\mathrm{mm})$ 进行清洗. 将 ITO 导电玻璃坚直放置人盛有 $\mathrm{SiO}_{2}$ 胶体球悬浮液(溶剂为无水乙醇)的烧杯里. 将烧杯置 人恒温的培养箱中, 设定温度为 $30^{\circ} \mathrm{C}$. 大约两天后, 乙醇溶剂完全挥发, 就制备出了 $\mathrm{SiO}_{2}$ 光子晶体薄膜.

(iv) $\mathrm{SiO}_{2}-\mathrm{ZnO}$ 复合光子晶体薄膜的制备. 在本 文中, 我们采用电沉积方法和烧结处理制备 $\mathrm{SiO}_{2}-\mathrm{ZnO}$ 复合光子晶体. 电沉积装置采用标准三电 极沉积槽, 以铂电极为对电极, 饱和甘录电极为参比 电极, 工作电极是附有 $\mathrm{SiO}_{2}$ 光子晶体薄膜的 ITO 导 电玻璃, 电解液为 $0.1 \mathrm{~mol} \cdot \mathrm{L}^{-1} \mathrm{Zn}(\mathrm{Ac})_{2}$ 水溶液. 工作 电压设定为 $-1.0 \mathrm{~V}$, 沉积时间分别为 $5,10,20 \mathrm{~min}$.
在空气气氛下将电沉积所得到的薄膜在 $500^{\circ} \mathrm{C}$ 下烧 结处理 $2 \mathrm{~h}$ 得到 $\mathrm{SiO}_{2}-\mathrm{ZnO}$ 复合光子晶体薄膜.

\section{2 结果与讨论}

\section{$2.1 \mathrm{SiO}_{2}$ 光子晶体模板的形貌表征}

图 1(a)是垂直沉积所制备的 $\mathrm{SiO}_{2}$ 光子晶体模板 的表面形貌. 从图中可以看到, 平均直径为 $250 \mathrm{~nm}$ 的 $\mathrm{SiO}_{2}$ 微球紧密堆积, 即在每一层中, 任 1 个 $\mathrm{SiO}_{2}$ 微球都与其余的 6 个 $\mathrm{SiO}_{2}$ 微球相接触. 尽管很难区 分这种堆积结构为面心立方 (fcc)结构还是六角密堆 (hcp)结构, 但是理论计算表明 fcc 结构更稳定 ${ }^{[15]}$. 在 fcc 堆积方式下, 每一层都与基底平行并沿着(111)轴 层层堆积, 在这个组装过程中产生了一些内生缺陷 如线缺陷与点缺陷. 图 1(b)是光子晶体模板的截面图, 图中显示所制备的 $\mathrm{SiO}_{2}$ 光子晶体模板其厚度约 $11 \mu \mathrm{m}$.

\section{$2.2 \mathrm{SiO}_{2}-\mathrm{ZnO}$ 复合光子晶体薄膜形貌}

图 2(a), (b) 是在沉积电压为 $-1.0 \mathrm{~V}$, 沉积时间为 5 $\min$ 所制备的 $\mathrm{SiO}_{2}-\mathrm{ZnO}$ 复合光子晶体断面 $\mathrm{SEM}$ 图片, 因沉积时间较短, $\mathrm{ZnO}$ 颗粒随机黏附在 $\mathrm{SiO}_{2}$ 微球表面, 而且 $\mathrm{ZnO}$ 颗粒并不相同.
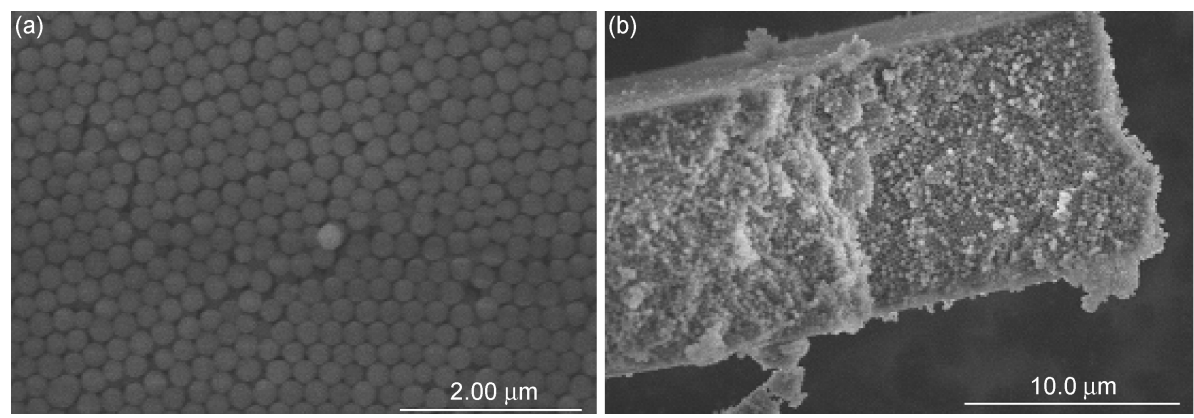

图 $1 \mathrm{SiO}_{2}$ 光子晶体模板的 $\mathrm{SEM}$ 照片 (a) 表面形貌; (b) 截面形貌
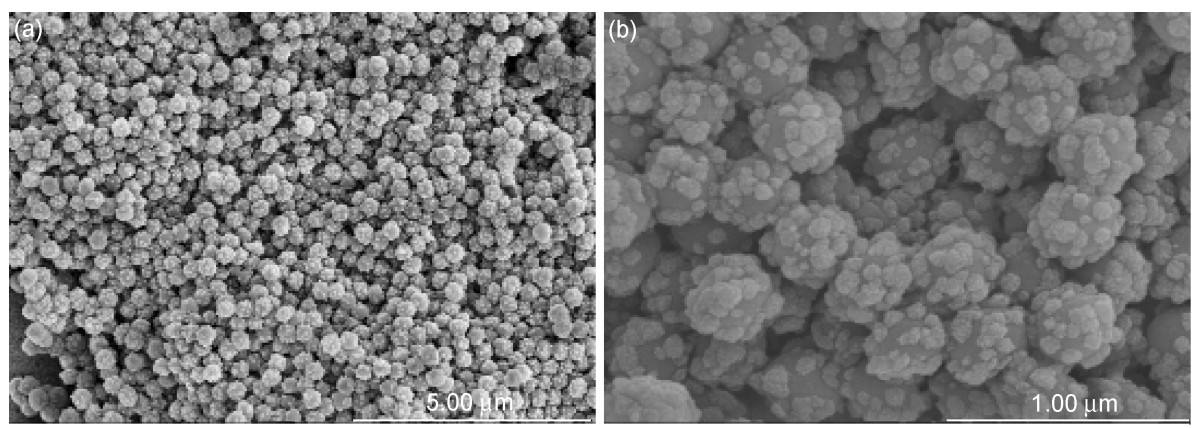

图 2 电沉积 $5 \mathrm{~min}$ 所制备的 $\mathrm{SiO}_{2}-\mathrm{ZnO}$ 复合光子晶体 $\mathrm{SEM}$ 图片 
图 3(a), (b) 是在沉积电压为 $-1.0 \mathrm{~V}$, 沉积时间为 $10 \mathrm{~min}$ 所制备的 $\mathrm{SiO}_{2}-\mathrm{ZnO}$ 复合光子晶体表面形貌图. 从图中可知, 尽管存在一些线状的 $\mathrm{ZnO}$ 物质依附生 长于 $\mathrm{SiO}_{2}$ 微球缝隙当中, 大部分 $\mathrm{ZnO}$ 物质均匀地包 覆在 $\mathrm{SiO}_{2}$ 微球表面.

图 4(a) (c) 分别是在沉积电压为 $-1.0 \mathrm{~V}$, 沉积时 间为 $20 \mathrm{~min}$ 所制备的 $\mathrm{SiO}_{2}-\mathrm{ZnO}$ 复合光子晶体表面和 截面形貌图. 从图中可知 $\mathrm{SiO}_{2}$ 光子晶体模板的缝隙 完全被 $\mathrm{ZnO}$ 物质填满.

根据上述观察结果, 可知在电沉积过程中, 由于 $\mathrm{Zn}$ 离子带正电荷, 当对工作电极加一负电压时, 电 解液中的 $\mathrm{Zn}$ 离子向 $\mathrm{SiO}_{2}$ 光子晶体模板移动, 从 ITO 导电玻璃发出的电子在 $\mathrm{SiO}_{2}$ 光子晶体模板的缝隙中
运动并与 $\mathrm{Zn}$ 离子结合, 这样当电沉积时间很短时, $\mathrm{Zn}$ 颗粒就随机生长在 $\mathrm{SiO}_{2}$ 微球表面. 随着沉积时间 的增长, $\mathrm{Zn}$ 颗粒完全包覆 $\mathrm{SiO}_{2}$ 微球, 最后完全填满 $\mathrm{SiO}_{2}$ 光子晶体模板的缝隙. 对电沉积所制备的薄膜 进行烧结处理就得到了 $\mathrm{SiO}_{2}-\mathrm{ZnO}$ 复合光子晶体. 在 电沉积过程中, 由于 $\mathrm{Zn}$ 离子远远小于 $\mathrm{Zn}$ 纳米颗粒, 所以 $\mathrm{Zn}$ 能够随机生长在所有的 $\mathrm{SiO}_{2}$ 微球表面. 因而 电沉积制备方法避免了电泳沉积制备复合光子晶体 时出现的 $\mathrm{SiO}_{2}$ 光子晶体表面纳米通道堵塞的现象 ${ }^{[12]}$.

\section{3 光学性能分析}

蛋白石结构的光子晶体其带隙位置可根据 Bragg 定律计算:

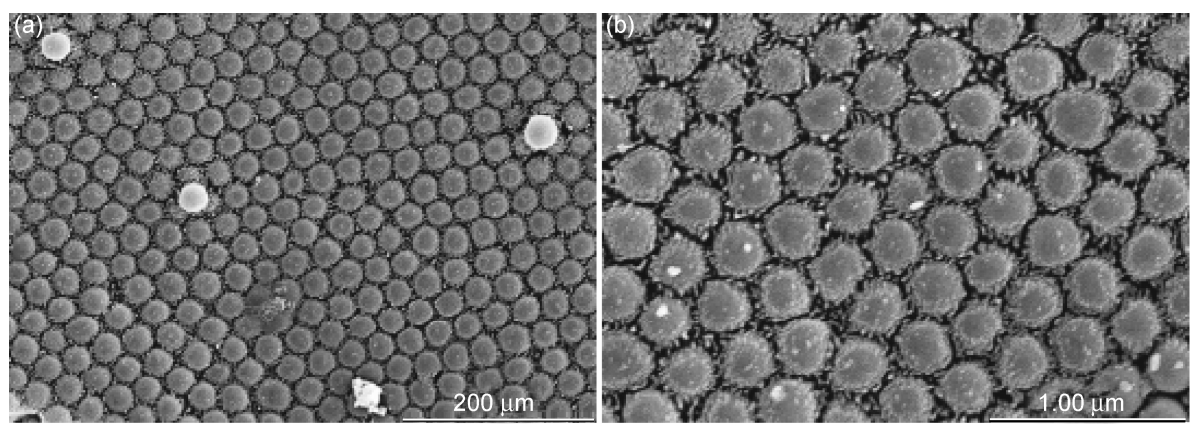

图 3 电沉积 $10 \mathrm{~min}$ 所制备的 $\mathrm{SiO}_{2}-\mathrm{ZnO}$ 复合光子晶体 $\mathrm{SEM}$ 图片
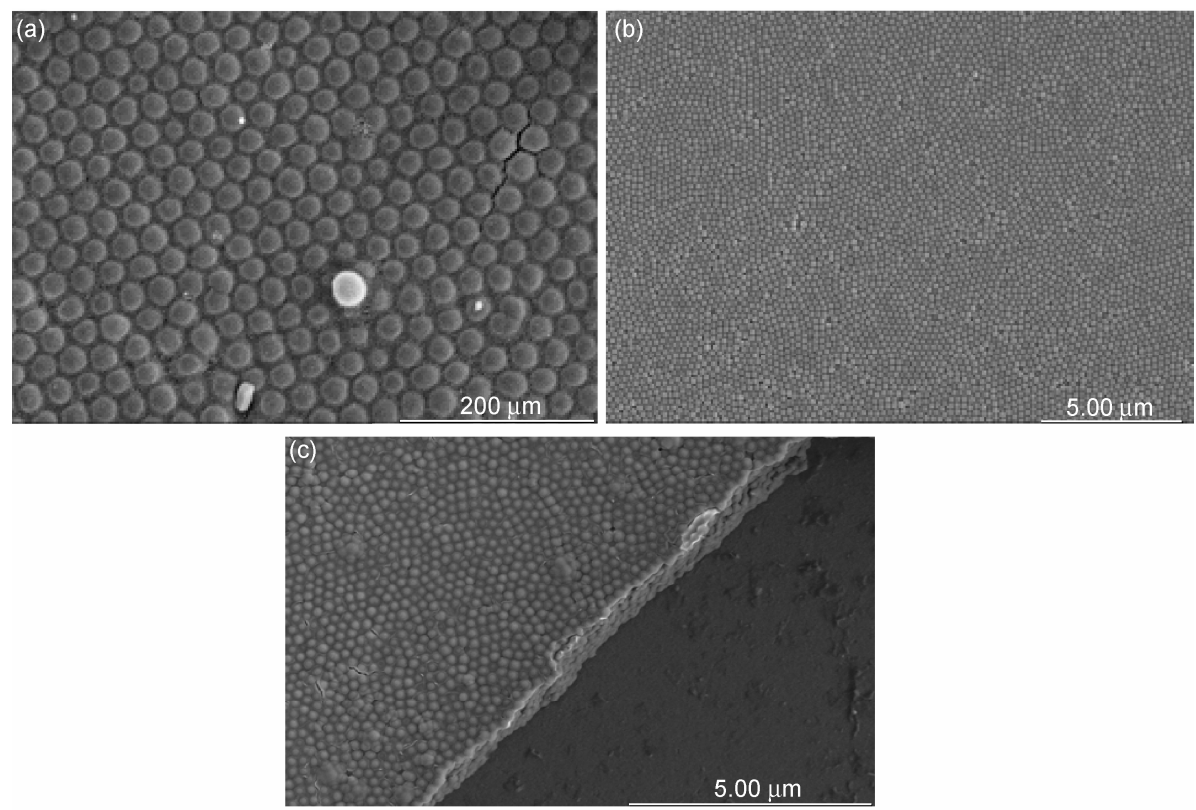

图 4 电沉积 $20 \mathrm{~min}$ 所制备的 $\mathrm{SiO}_{2}-\mathrm{ZnO}$ 复合光子晶体 $\mathrm{SEM}$ 图片 


$$
\begin{gathered}
\lambda_{\text {max }}=2 d_{111} \sqrt{n_{\text {avg }}^{2}-\sin ^{2} \theta}, \\
n_{\text {avg }}=\sqrt{n_{c}^{2} f+n_{v}^{2}(1-f)},
\end{gathered}
$$

式中, $d_{111}$ 为(111)面的相邻晶面间距; $n_{\mathrm{avg}}$ 是光子晶体 结构的有效折射率, $n_{c}$ 为胶体微球的折射率, $n_{v}$ 为空 隙或填充材料的折射率; $f$ 为填充系数 (本实验中, $f=74 \%$ ); $\theta$ 为人射光与样品表面法线夹角(本实验中, $\theta$ 为 0 ), 对于 FCC 结构, 晶面间距 $d_{111}=\sqrt{2} / 3 D=0.816 D$, $D$ 为胶体微球直径.

图 5 是 $\mathrm{SiO}_{2}$ 光子晶体模板与不同沉积时间所制 备的 $\mathrm{SiO}_{2}-\mathrm{ZnO}$ 复合光子晶体的反射光谱图. 定义 $\Delta \lambda$ 为反射峰的半高宽, $\lambda$ 为光子带隙的位置. 通过比较 光子晶体的反射峰峰高 $(h(\%))$ 和反射峰的相对带宽 $(\Delta \lambda / \lambda)$ 来评定光子晶体光学性能的优劣: 光子晶体结 构中的一些缺陷可以导致一个低的反射峰并产生反 射峰的宽化效应 ${ }^{[16,17]}$. 反射峰越高, 相对带宽值越小 表明所制备的光子晶体薄膜质量优异, $\mathrm{ZnO}$ 物质在 $\mathrm{SiO}_{2}$ 微球表面及 $\mathrm{SiO}_{2}$ 光子晶体模板缝隙中生长越均匀.

从表 1 中可得出如下结论: (1) 所有制备的光子 晶体在薄膜的法线方向上都出现了光子带隙, (2) 其 光子带隙的位置随着沉积时间的变化而变化. $\mathrm{SiO}_{2}$ 光 子晶体模板和 $20 \mathrm{~min}$ 电沉积制备的 $\mathrm{SiO}_{2}-\mathrm{ZnO}$ 复合光 子晶体其光子带隙位置的实验测定值与由 Bragg 定 律得出的计算值相吻合. 较短时间沉积的 $\mathrm{SiO}_{2}-\mathrm{ZnO}$ 复合光子晶体, 其反射峰的峰高值较小, 而相对带宽 值较大. 这是由于在很短沉积时间内, 随机附着在 $\mathrm{SiO}_{2}$ 微球表面的 $\mathrm{ZnO}$ 颗粒破坏了蛋白石光子晶体结 构的有序性. 随着沉积时间的增加, 由于 $\mathrm{ZnO}$ 物质 完全填满 $\mathrm{SiO}_{2}$ 光子晶体模板的缝隙, 提高了 $\mathrm{SiO}_{2}-\mathrm{ZnO}$ 复合光子晶体结构的有序性, 因而其反射 峰的峰高增高, 相对带宽值变小. 上述光学性能的分 析结果与前面 SEM 结果是一致的.

\section{3 结论}

综上所述, 我们采用电沉积法和烧结处理成功 制备出了 $\mathrm{SiO}_{2}-\mathrm{ZnO}$ 复合光子晶体. 并研究了电沉积

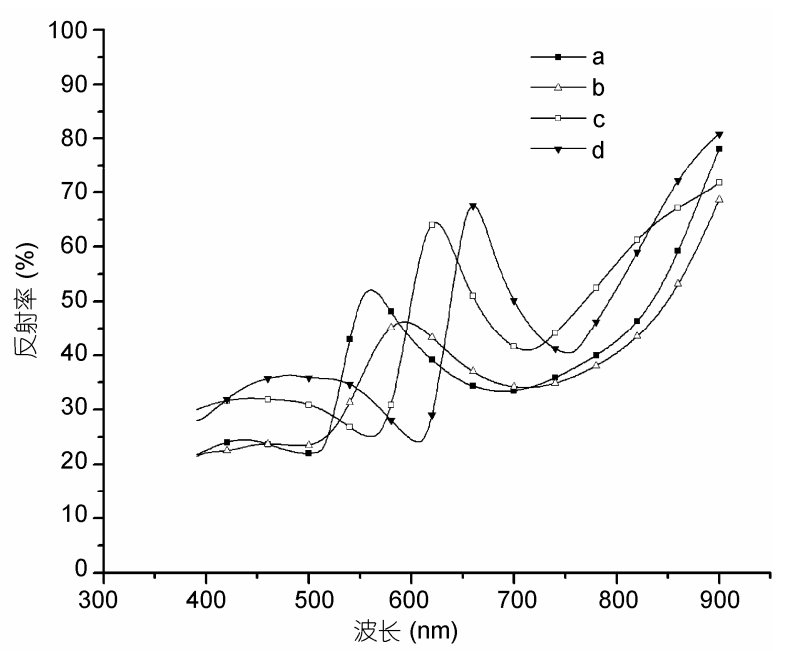

图 $5 \mathrm{SiO}_{2}$ 光子晶体模板与不同沉积时间所制备的 $\mathrm{SiO}_{2}-\mathrm{ZnO}$ 复合光子晶体的反射光谱

(a) $\mathrm{SiO}_{2}$ 光子晶体模板; (b) $5 \mathrm{~min}$ 电沉积所制备 $\mathrm{SiO}_{2}-\mathrm{ZnO}$ 复合光子晶 体; (c) $10 \mathrm{~min}$ 电沉积所制备 $\mathrm{SiO}_{2}-\mathrm{ZnO}$ 复合光子晶体; (d) $20 \mathrm{~min}$ 电沉 积所制备 $\mathrm{SiO}_{2}-\mathrm{ZnO}$ 复合光子晶体

表 $1 \mathrm{SiO}_{2}$ 光子晶体模板及不同沉积时间所制备 $\mathrm{SiO}_{2}-\mathrm{ZnO}$ 复合光子晶体的光学性质表征 ${ }^{\text {a) }}$

\begin{tabular}{ccccc}
\hline 光学性质 & $\lambda_{\mathrm{C}} / \mathrm{nm}$ & $\lambda_{\mathrm{m}} / \mathrm{nm}$ & $\Delta \lambda / \lambda(\%)$ & $h(\%)$ \\
\hline $\mathrm{SiO}_{2}$ 光子晶体模板 & 556 & 560 & 13.5 & 23.7 \\
$5 \mathrm{~min}$ 分钟电沉积所制 & - & 593 & 17.1 & 17.3 \\
$\quad \begin{array}{c}\text { 备 } \mathrm{SiO}_{2}-\mathrm{ZnO} \text { 薄膜 } \\
10 \mathrm{~min} \text { 电沉积所制备 }\end{array}$ & - & 624 & 11.1 & 21.1 \\
$\begin{array}{c}\mathrm{SiO}_{2}-\mathrm{ZnO} \text { 薄膜 } \\
20 \mathrm{~min} \text { 电沉积所制备 } \\
\mathrm{SiO}_{2}-\mathrm{ZnO} \text { 薄膜 }\end{array}$ & 676 & 660 & 9.5 & 32.3 \\
\hline
\end{tabular}

a) $\lambda_{\mathrm{c}}$, 光子带隙理论计算位置; $\lambda_{\mathrm{m}}$, 光子带隙实验值; $\Delta \lambda / \lambda$, 反射峰 的相对带宽(实验值); $h$, 反射峰峰高

时间对 $\mathrm{SiO}_{2}-\mathrm{ZnO}$ 复合光子晶体的形貌及光学性 能的影响. 研究发现, 当电沉积时间较短时, $\mathrm{ZnO}$ 颗 粒随机附着在 $\mathrm{SiO}_{2}$ 微球表面, 所制备的 $\mathrm{SiO}_{2}-\mathrm{ZnO}$ 复 合光子晶体其光反射性能较差. 随着沉积时间的增 长, $\mathrm{ZnO}$ 物质完全包覆 $\mathrm{SiO}_{2}$ 微球进而填满 $\mathrm{SiO}_{2}$ 光子 晶体模板缝隙, 所制备出的 $\mathrm{SiO}_{2}-\mathrm{ZnO}$ 复合光子晶体 具有良好的光反射性能. 此外, 电沉积制备方法还避 免了其他制备方法所出现的表面纳米通道堵塞现象.

\section{参考文献}

1 John S. Strong localization of photons in certain disordered dielectric superlattices. Phys Rev Lett, 1987, 58: 2486-2489

2 Yablonovitch E. Inhibited spontaneous emission in solid-state physics and electronics. Phys Rev Lett, 1987, 58: 2059-2062

3 Van Blaaderen A, Ruel R, Wiltzius P. Template-directed colloidal crystallization. Nature, 1997, 385: 321-324 
4 Jiang P, Bertone J F, Hwang K S, et al. Single-crystal colloidal multilayers of controlled thickness. Chem Mater, 1999, 11: 2132-2140

5 陈金金, 孙志强, 陈志民, 等. 双基片垂直沉积法制备稳定的胶体晶体晶片. 科学通报, 2005, 50: 321一-326

6 Jiang P, Ostojic G N, Narat R, et al. The fabrication and bandgap engineering of photonic multilayers. Adv Mater, 2001, 13: 389-393

7 Holgado M, Garcia-Santamaria F, Blanco A, et al. Electrophoretic deposition to control artificial opal growth. Langmuir, 1999, 15: 47014704

8 方俊, 王秀峰, 王列松, 等. 丁二酸改性二氧化硅胶体球的制备及其胶体晶体的组装. 科学通报, 2006, 51: 2842一 2846

9 Teh L K, Yeo K H, Wong C C. Isotropic photonic pseudogap in electrodeposited ZnO inverse opal. Appl Phys Lett, 2006, 89: 051105051107

10 Mine E, Hirose M, Kubo M, et al. Synthesis of submicron-sized titania-coated silica particles with a sol-gel method and their application to colloidal photonic crystals. J Sol-Gel Sci Technol, 2006, 38: 91-95

11 Waterhouse G I N, Waterland M R. Opal and inverse opal photonic crystals: Fabrication and characterization. Polyhedron, 2007, 26: 356368

12 Chung Y W, Leu I C, Lee J H, et al. Filling behavior of ZnO nanoparticles into opal template via electrophoretic deposition and the fabrication of inverse opal. Electrochim Acta, 2009, 54: 3677-3682

13 Abrarov S M, Yuldashev S U, Kim T W, et al. Effect of photonic band-gap on photoluminescence of ZnO deposited inside the green synthetic opal. Opt Commun, 2005, 250: 111-119

14 Stöber W, Fink A, Bohn E. Controlled growth of monodisperse silica spheres in micron size range. J Colloid Interface Sci, 1968, 26: 6269

15 Woodcock L V. Entropy difference between the face centred cubic packed and hexagonal closed packed crystal structure. Nature, 1997, 385: $141-143$

16 Piret F, Su B L. Effects of pH and ionic strength on the self-assembly of silica colloids to opaline photonic structures. Chem Phys Lett, 2008, 457: 376-380

17 Rengarajan R, Mittleman D. Effect of disorder on the optical properties of colloidal crystals. Phys Rev E, 2005, 71: 016615-016625 